\title{
Fluid flow-induced activation of subcellular AMPK and its interaction with FAK and Src
}

\author{
Yunxia Guo ${ }^{1,2}$, Hannah E Steele ${ }^{1}$, Bai-Yan $\mathrm{Li}^{2 *}$, Sungsoo $\mathrm{Na}^{1 *}$ \\ ${ }^{1}$ Department of Biomedical Engineering, Indiana University Purdue University Indianapolis, \\ Indianapolis, IN 46202, USA \\ ${ }^{2}$ Department of Pharmacology, School of Pharmacy, Harbin Medical University, \\ Harbin 150081, China
}

Keywords: mechanotransduction; metabolic signaling; AMPK; Src; FAK

Running title: Mechanotransduction of subcellular AMPK

Abbreviations:

AMPK: AMP-activated protein kinase

FAK: focal adhesion kinase

IFF: interstitial fluid flow

FRET: fluorescence resonance energy transfer

CFP: cyan fluorescent protein

YFP: yellow fluorescent protein

FHA1: forkhead-associated domain 1

* Co-corresponding Authors:

Sungsoo $\mathrm{Na}, \mathrm{PhD}$

Department of Biomedical Engineering

Indiana University Purdue University Indianapolis

723 W. Michigan Street, SL220H

Indianapolis, IN 46202, USA

Phone: 317-278-2384

E-mail: sungna@iupui.edu

Bai-Yan Li, MD/PhD

Department of Pharmacology

School of Pharmacy, Harbin Medical University

\#157 Baojian Road, Harbin 150081, China

Phone/Fax: +86 451-8667-1354

E-mail: liby@ems.hrbmu.edu.cn

This is the author's manuscript of the article published in final edited form as:

Guo, Y., Steele, H. E., Li, B.-Y., \& Na, S. (2020). Fluid flow-induced activation of subcellular AMPK and its interaction with

FAK and Src. Archives of Biochemistry and Biophysics, 679, 108208. https://doi.org/10.1016/j.abb.2019.108208 


\begin{abstract}
AMP-activated protein kinase (AMPK) is a metabolic energy sensor that plays a critical role in cancer cell survival and growth. While the physical microenvironment is believed to influence tumor growth and progression, its role in AMPK regulation remains largely unknown. In the present study, we evaluated AMPK response to mechanical forces and its interaction with other mechano-responsive signaling proteins, FAK and Src. Using genetically encoded biosensors that can detect AMPK activities at different subcellular locations (cytosol, plasma membrane, nucleus, mitochondria, and Golgi apparatus), we observed that AMPK responds to shear stress in a subcellular location-dependent manner in breast cancer cells (MDA-MB-231). While normal epithelial cells (MCF-10A) also similarly responded to shear stress, they are less sensitive to shear stress compared to MDA-MB-231 cells. Inhibition of FAK and Src significantly decreased the basal activity level of AMPK at all five subcellular locations in MDA-MB-231 cells and selectively blocked shear stress-induced AMPK activation. Moreover, testing with cytoskeletal drugs revealed that myosin II might be the critical mediator of shear stress-induced AMPK activation in MDA-MB-231 cells. These findings suggest that breast cancer cells and normal epithelial cells may have different mechanosensitivity in AMPK signaling and that FAK and Src as well as the myosin II-dependent signaling pathway are involved in subcellular AMPK mechanotransduction in breast cancer cells.
\end{abstract}




\section{Introduction}

The physical tumor microenvironment affects tumor growth and progression[1-3]. As the cancer grows, accumulation and reorganization of extracellular matrix and abnormal growth of cancer cells result in a buildup of pressure and increased interstitial fluid flow (IFF) within the tumor tissue[4]. This IFF in turn generates shear stress to influence cancer cell invasion and proliferation possibly via mechanotransduction signaling pathways[5-7]. FAK and Src are such mechanotransduction signaling proteins whose activities are increased in cancer cells, thereby enhancing tumor growth and metastasis[8]. For example, activation of Src specifically enhances the ability of breast cancer cells to grown in bone marrow microenvironment and bone metastasis in breast cancer requires Src-dependent survival signals[9]. FAK promotes tumor progression and metastasis by controlling cell migration, invasion, survival, and cancer stem cell selfrenewal[10]. While these cellular energy-consuming processes involve Src or FAK, it is unclear whether cellular energy homeostasis is related to these signaling proteins.

AMPK regulates cellular energy homeostasis by sensing the ratios of AMP/ATP and ADP/ATP[11]. In addition to its major role in cellular energy sensing, AMPK has been shown to have multiple roles in cell functions including cell growth, protein synthesis, autophagy, and gene transcription[12]. It is increasingly recognized that AMPK's multiple, and sometimes paradoxical, roles in cell physiology as well as pathological diseases such as cancer may be due to its compartmentalized subcellular activation because compartmentalization is considered to be one of the properties that molecules utilize to conduct multi-tasking $[13,14]$. However, it is not clear whether distinct subcellular pools of AMPK respond to specific extracellular stimuli. While 
AMPK is known to be activated by metabolic stress, recent reports have demonstrated that it is also activated by mechanical forces, suggesting its involvement in mechanotransduction[15-17].

In this study, we used genetically encoded biosensors that are specific to subcellular locations to visualize compartmentalized AMPK signaling activities[13]. Fluid flow-induced shear stress was applied to two types of the cells, breast cancer cells and normal epithelial cells, to study subcellular AMPK responses to mechanical forces. Finally, the role of Src and FAK as well as the cytoskeletal components in the shear stress-induced AMPK signaling activities were explored to examine the involvement of mechanotransduction in AMPK activities.

\section{Materials and Methods}

\subsection{Biosensors and plasmids}

FRET-based biosensors for monitoring subcellular compartment-specific AMPK activities were kindly provided by Dr. Jin Zhang (University of California San Diego) and Dr. Takanari Inoue (Johns Hopkins University). Briefly, the AMPK biosensors are composed of CFP, the FHA1 domain, the AMPK substrate motif, and YFP. The phosphorylation of the AMPK substrate promotes its intramolecular binding to the FHA1 domain, which results in the close association of the donor (CFP) and acceptor (YFP) and subsequent FRET from the donor to the acceptor. Hence, AMPK activities can be visualized by the changes of the emission ratio of FRET/CFP. The different sequences were fused to well-established signal sequences to produce biosensors targeting various organelles including cytosol (Cyto-AMPK), plasma membrane (PM-AMPK), nucleus (Nuc-AMPK), mitochondria (Mito-AMPK), and Golgi apparatus (Golgi-AMPK)[13]. The specificity of these subcellular compartment-specific AMPK biosensors have been 
extensively tested and their localization has been confirmed using the specific markers[13, 18$]$. The Src and FAK biosensors were kindly provided by Dr. Yingxiao Wang (University of California San Diego). The Src biosensor contains the CFP, the SH2 domain from c-Src, the Src substrate peptide and YFP[19]. Similarly, the FAK biosensor consists of CFP, the SH2 domain, the FAK substrate peptide and YFP[20]. Because the plasma membrane is important in mechanotransduction of FAK and Src, we used the biosensors (Lyn-FAK and Lyn-Src) targeted to the plasma membrane with a lipid raft-targeting motif, derived from Lyn kinase[21]. The CFP/FRET emission ratio was used to measure the activity of Src and FAK.

\subsection{Cell culture and transfection}

MDA-MB-231, breast cancer cell lines, were cultured in Dulbecco's modified Eagle's medium (DMEM; Lonza) containing 10\% FBS (Hyclone) and 1\% penicillin/streptomycin (Lonza). MCF10A cells were cultured in Dulbecco's Modified Eagle Medium: Nutrient Mixture F-12 (DMEM/F12; Gibco) supplemented with 10\% FBS (Hyclone), 1\% penicillin/streptomycin (Lonza), hydrocortisone $(50 \mu \mathrm{g} / \mathrm{ml})$, insulin $(20 \mu \mathrm{g} / \mathrm{ml})$, and hEGF (200 ng/ml). For imaging experiments, cells were plated on a type I collagen-coated glass bottom dish (MatTek) or $\mu$-slide cell culture chamber (Ibidi), and then transfected with one of the FRET-based biosensors using Lipofectamine LTX (Invitrogen) according to the manufacturer's protocol. All the cells were maintained at $37^{\circ} \mathrm{C}$ and $5 \% \mathrm{CO}_{2}$ in a humidified incubator prior to experiments.

\subsection{Chemical reagents and siRNAs}

PP2 (Sigma; 0.1, 1, $10 \mu \mathrm{M}$ ) was used to inhibit Src activities, and PF-573228 (Sigma; 10, 100, $1000 \mathrm{nM}$ ) was used to inhibit FAK activities. Cytochalasin D (Enzo Life Sciences; $100 \mathrm{ng} / \mathrm{ml}$ ) 
was used to disrupt actin filaments. Nocodazole (Sigma; $1 \mu \mathrm{M}$ ) was used to inhibit microtubules. Blebbistatin (Toronto Research Chemicals; $50 \mu \mathrm{M}$ ) was used to inhibit myosin II. ML-7 (Biomol; $20 \mu \mathrm{M}$ ) was used to inhibit myosin light chain kinase. Compound C (Sigma, $10 \mu \mathrm{M}$ ) and A769662 (Tocris, $25 \mu \mathrm{M}$ ) were used as an AMPK inhibitor and activator, respectively. FAK siRNA, Src siRNA, and non-specific control (NC) siRNA were obtained from Santa Cruz Biotechnology.

\subsection{Shear stress application}

In this study, a unidirectional flow was applied to the cells grown in the $\mu$-slide cell culture chamber (Ibidi). We used 2.5 and 10 dynes $/ \mathrm{cm}^{2}$ of the shear stress, which was regulated by controlling the flow rate of a peristaltic pump (Cole-Parmer). During shear stress application, the chamber was perfused with HEPES-buffered, phenol red-free DMEM without serum to maintain the $\mathrm{pH}$ at 7.4. The shear stress application experiments were conducted at $37^{\circ} \mathrm{C}$ by using a temperature-controlled air blower (Nevtek).

\subsection{Microscopy and image analysis}

Images were obtained by using a Nikon Ti-E inverted microscope equipped with an Evolve 512 electron-multiplying charge-coupled device (EMCCD) camera (Photometrics), a filter wheel controller (Sutter Instruments), and a Perfect Focus System (Nikon) that maintains the focus during time-lapse imaging. The following filter sets (Semrock) were used for FRET imaging: CFP excitation: 438/24 (center wavelength/bandwidth in nm); CFP emission: 483/32; YFP (FRET) emission: 542/27. To minimize photobleaching, a neutral density (ND) 32 filter was used. Time-lapse images were recorded every 2 minutes with a $40 \times$ objective with 0.75 
numerical aperture. FRET images for AMPK, Src, and FAK activity were generated with NISElements software (Nikon). The FRET ratio images were scaled and represented according to the color bar.

\subsection{Statistical analysis}

Statistical analyses were conducted using Prism 5 software (GraphPad). Statistical data are presented as the mean \pm standard error of the mean (SEM). One-way ANOVA followed by Dunnett's post hoc test was used to determine the statistical differences among multiple groups, and the differences between two groups were evaluated with the two-tailed Student's $t$-test. A $p$ value less than 0.05 was considered statistically significant.

\section{Results}

\subsection{Subcellular AMPK response to shear stress in MDA-MB-231 cells}

Several studies have recently reported that AMPK responds to mechanical forces[15, 16]. However, little is known about its subcellular response at the single-cell level. Thus, we sought to visualize AMPK activities at different subcellular organelles in response to fluid flow-induced shear stress. We transfected MDA-MB-231 cells with one of the five FRET-based AMPK biosensors: Cyto-AMPK, PM-AMPK, Nuc-AMPK, Mito-AMPK, and Golgi-AMPK targeting cytosol, plasma membrane, nucleus, mitochondria, and Golgi apparatus, respectively. The AMPK activities were measured by monitoring changes of the FRET/CFP emission ratios of the biosensors in the same cell. We observed that shear stress-induced AMPK activities and their mechanosensitivity were organelle dependent. (Fig. 1a-e). Cyto-AMPK activities were rapidly enhanced by both low (37\% increase in $4 \mathrm{~min})$ and high shear stresses (51.4\% increase in $2 \mathrm{~min})$. 
PM-AMPK activities were differently regulated by shear stresses. Under high shear stress, its activities were substantially increased (36.8\% at $60 \mathrm{~min})$, but under low shear stress, its activities were significantly decreased (24.2\% at $60 \mathrm{~min})$. Nuc-, Mito-, and Golgi-AMPK activities were also upregulated by high shear stress, but not significantly affected by low shear stress. As a control, we conducted FRET imaging under shear stress at 0 dyne $/ \mathrm{cm}^{2}$, and it did not significantly affect AMPK activities in MDA-MB-231 cells (Supplementary Fig. S1a). These results indicate that in MDA-MB-231 cells AMPK is activated by shear stress and its activities are dependent on the subcellular locations.

\subsection{Subcellular AMPK response to shear stress in MCF-10A cells}

Many lines of evidence suggest that deregulated control of cell population observed in tumor is due to the cancer cell's altered energy metabolism[22]. In this study, we hypothesized that AMPK response to shear stress and its activation level in breast cancer cells (MDA-MB-231) and normal breast epithelial cells (MCF-10A) might be different. To test his hypothesis, we transfected MCF-10A cells with one of the AMPK biosensors and observed AMPK activities under shear stress. The results revealed that in MCF-10A cells, Cyto-AMPK activities were highly responsive to shear stress, and the activation levels were similar to those of MDA-MB231 under both low and high shear stresses (Fig. 2a). However, PM-AMPK and Mito-AMPK in MCF-10A cells did not significantly respond to shear stress (Fig. 2b, d). They were initially activated by shear stress but returned to the untreated basal levels in 20 minutes. The activation pattern and level were significantly different from those observed in MDA-MB-231 (Fig. 1b, d). These results suggest that unlike MDA-MB-231 cells, AMPK at the plasma membrane and mitochondria is not mechanoresponsive in MCF-10A cells. Nuc-AMPK and Golgi-AMPK were 
substantially activated by high shear stress (Fig. 2c, e) although to a lesser degree compared to the shear stress response in MDA-MB-231 cells (Fig. 1c, e). They did not significantly respond to low shear stress, similar to the response in MDA-MB-231 cells. A shear stress at 0 dyne $/ \mathrm{cm}^{2}$ did not significantly affect AMPK activities in MCF-10A cells (Supplementary Fig. S1b). Taken together, these results indicate that in response to shear stress, AMPK activity in the specific subcellular locations (i.e., plasma membrane and mitochondria) might differently respond to shear stress in MDA-MB-231 and MCF-10A cells, suggesting the distinct roles of the subcellular location-specific AMPK activation in MDA-MB-231 and MCF-10A cells.

\subsection{Distinct involvement of FAK and Src in subcellular AMPK activities in MDA-MB 231 and MCF-10A cells}

Our results on AMPK response to shear stress suggest that AMPK might be linked to the mechanotransduction signaling pathway such as FAK and Src[23-25]. To test this hypothesis, we transfected cells with one of the five AMPK biosensors, and the cells were imaged for 1hour under the treatment of FAK inhibitor PF573228 $(10,100,1000 \mathrm{nM})$ or Src inhibitor PP2 (0.1, 1 , $10 \mu \mathrm{M})$. The highest concentrations of PF573228 $(1000 \mathrm{nM})$ and PP2 $(10 \mu \mathrm{M})$ used in this study have been reported to effectively inhibit FAK and Src, respectively[26-30]. In MDA-MB-231 cells, AMPK activities in all subcellular locations were significantly decreased under $1000 \mathrm{nM}$ of PF573228 (Fig. 3a). Under lower concentrations of PF573228 (10 and 100 nM), only CytoAMPK activities were significantly reduced, while other subcellular AMPK activities were not significantly altered (data not shown). AMPK activities were also significantly decreased in all subcellular locations under $10 \mu \mathrm{m}$ of PP2 (Fig. 3b), while they were not altered under lower concentrations of PP2 (data not shown). We further confirmed the effects of FAK or Src 
inhibition on AMPK activity using siRNAs (Supplementary Fig. S2). The results showed similar inhibitory effects as compared to those by the specific drugs. In contrast to MDA-MB-231 cells, MCF-10A cells showed selective suppression of subcellular AMPK under PF573228 and PP2 (Fig. 3c, d). AMPK activities at the cytosol, nucleus, and Golgi apparatus were significantly decreased by $1000 \mathrm{nM}$ of PF573228 (Fig. 3c), while those at other locations were not significantly altered. In response to $10 \mu \mathrm{M}$ PP2, only the cytosol and nucleus AMPK were downregulated, and the Golgi-AMPK was not altered (Fig. 3d). Under lower concentrations of the drugs subcellular AMPK activities were not altered (data not shown). The results in Fig. 3 on the global inhibition of AMPK in MDA-MB-231 as well as its selective suppression in MCF10A under FAK and Src inhibitors are consistent with shear stress-induced subcellular activation of AMPK (Figs. 1 and 2), suggesting the distinct role of FAK/Src-mediated mechanotransduction in subcellular AMPK activation in MDA-MB-231 and MCF-10A cells.

\subsection{Selective blockage of shear stress-induced AMPK activation by inhibition of FAK and Src}

To further investigate the significance of shear stress-induced AMPK mechanotransduction, we tested whether inhibition of FAK and Src blocks shear stress-induced subcellular AMPK activation. Cells transfected with one of the AMPK biosensors were pretreated with $1000 \mathrm{nM}$ PF573228 or $10 \mu \mathrm{M}$ PP2 for $1 \mathrm{~h}$ prior to FRET imaging. During imaging, cells were subjected to 10 dyne $/ \mathrm{cm}^{2}$ shear stress for $1 \mathrm{~h}$. The results revealed that in MDA-MB-231 cells, inhibition of FAK and Src by PF573228 and PP2 completely blocked shear stress-induced AMPK activities at the plasma membrane, nucleus, and mitochondria, whereas the inhibition did not have a significant effect on the shear stress-induced Cyto- and Golgi-AMPK activities (Fig. 4a). MCF- 
10A cells showed a different response (Fig. 4b). Although PF573228 and PP2 appeared to partially block shear stress-induced AMPK activities in Cyto-AMPK, they failed to completely block the activities. Golgi-AMPK in MCF-10A cells was also not affected by the two drugs. Consistent with the results shown in Figs 2 and 3, PF573228 and PP2 did not affect shear stressinduced PM- and Mito-AMPK. Only shear stress-induced Nuc-AMPK activities were completely blocked by PF573228 and PP2 in MCF-10A cells.

\subsection{The role of the cytoskeleton in the regulation of shear stress-induced AMPK activities}

The cytoskeleton and its associated activity have been shown to serve as a critical mediator for cellular mechanotransduction[23,31]. Thus, we next sought to address the mechanism by which they affect shear stress-induced AMPK activity. Cells expressing one of the AMPK biosensors were pretreated for 1 hour with one of the reagents that specifically disrupt or inhibit actin filaments (cytochalasin D), microtubules (nocodazole), myosin light chain kinase (ML-7), or myosin II activity (blebbistatin). During FRET imaging, shear stress was applied to the cells. Significant suppression of the shear stress-induced Cyto-AMPK activation was observed in both MDA-MB-231 and MCF10A cells pretreated with cytochalasin D, nocodazole, ML-7, and blebbistatin (Fig. 5a). Interestingly, shear stress-induced PM-AMPK activation was not altered by cytochalasin D, while other drugs significantly suppressed the activation in MDA-MB-231 cells (Fig. 5b). We observed that PM-AMPK in MCF-10A cells did not respond to shear stress (Fig. 2b). Similarly, none of the cytoskeletal drugs failed to alter its activation under shear stress

(Fig. 5b). Shear stress-induced Nuc-AMPK in both cell types was substantially decreased by all of the cytoskeletal drugs used in this study (Fig. 5c). In MDA-MB-231 cells, Mito-AMPK was significantly activated by shear stress and this activation was suppressed by cytochalasin D, 
nocodazole, ML-7, and blebbistatin (Fig. 5d). In MCF-10A cells, however, Mito-AMPK was not significantly altered by shear stress and other cytoskeletal drugs (Fig. 5d). Although all cytoskeletal drugs suppressed shear stress-induced Golgi-AMPK activation in MDA-MB-231 cells, ML-7 and blebbistatin appeared to completely block the activation (Fig. 5e). The cytoskeletal drugs did not affect shear stress-induced activation of Golgi-AMPK in MCF-10A cells (Fig. 5e). These results suggest the potentially distinct role of the cytoskeleton and its associated activity in subcellular AMPK activation.

\subsection{The role of AMPK in the activities of FAK and Src}

AMPK has been reported to negatively regulate integrin $\beta 1[32,33]$. Since integrin $\beta 1$ plays a critical role in FAK- and Src-mediated mechanotransduction[8, 23, 34], we sought to test the role of AMPK in FAK and Src activation. First, we measured the basal level of FAK and Src in response to AMPK activation and inhibition. Cells expressing the FAK or Src biosensor were treated with either $25 \mu \mathrm{M}$ A769662 (an AMPK activator) or $10 \mu \mathrm{M}$ Compound C (an AMPK inhibitor) for $1 \mathrm{~h}$ and then the basal levels of FAK and Src activation were compared with the non-treated control group. The results revealed that in MDA-MB-231 cells, the basal levels of both FAK and Src were substantially reduced by AMPK activation, while they were enhanced by AMPK inhibition (Fig. 6a). Similarly, MCF-10A cells showed a significant inhibition of the basal level of FAK and Src under AMPK activation. However, AMPK inhibition did not alter their basal levels in MCF-10A cells (Fig. 6b). Next, we tested the role of AMPK in the mechanotransduction of FAK and Src. Shear stress significantly increased FAK activity in both MDA-MB-231 and MCF-10A cells and this FAK mechanotransduction was not significantly affected by pretreatment of A769662 and Compound C (Fig. 6c). Src activity was also enhanced 
by shear stress in both MDA-MB-231 and MCF-10A cells (Fig. 6d). Interestingly, while pretreatment of A769662 did not affect Src mechanotransduction, Compound C completely blocked it (Fig. 6d). These results indicate that AMPK affects the basal level of FAK and Src activation, but it does not significantly affect shear stress-induced activation of FAK and Src.

\section{Discussion}

In this study, we used the subcellular compartment-specific AMPK biosensors to explore mechanotransduction of subcellular AMPK and its interaction with FAK and Src. The aim of this study is to visualize subcellular compartment-specific AMPK activity with high spatial and temporal precision using FRET-based biosensors. It has been demonstrated that the FRET-based biosensors allow for monitoring, in real time, phosphorylation of proteins in living cells, without the need to lyse cells for biochemical characterization[35, 36]. In recent reports describing the development of the AMPK FRET biosensor showed that the AMPK activity measured by the FRET biosensor was specific to AMPK, and similar to that measured by phosphorylation status[13, 37]. We showed that AMPK responds to mechanical forces, and that its subcellular compartment-specific responses are different between breast cancer cells and normal epithelial cells. Several studies demonstrated mechanical force-induced AMPK activation. For example, in vascular endothelial cells, shear stress-induced AMPK activation is shown to regulate vascular homeostasis by increasing eNOS (endothelial nitric oxide synthase; vasodilator) and decreasing ET-1 (endothelin 1; vasoconstrictor)[16]. ATP is released by shear stress to mediate eNOS in endothelial cells [38]. In epithelial cells, mechanical force applied to E-cadherin induces AMPK activation which contributes to junction reinforcement via increased E-cadherin and F-actin enrichment at tight junctions[15]. These studies quantified the level of global AMPK activities in 
a group of cells. However, several lines of evidence indicate distinct subcellular localization of AMPK within a cell, such as nucleus[39] and mitochondria[17], and AMPK shuttling between nucleus and cytoplasm is influenced by environmental stresses such as heat shock or oxidant exposure[40]. Thus, we postulated that AMPK response to mechanical force may be dependent on subcellular location. In the present study, we observed that MDA-MB-231 and MCF-10A cells exhibit highly distinct subcellular AMPK activation patterns in response to fluid flow. Specifically, AMPK response at the plasma membrane and mitochondria was enhanced in MDAMB-231 cells, but not in MCF-10A cells under fluid flow. Little is known about the molecular mechanism of these distinct AMPK responses between cancer and normal cells under flow. Moreover, the role of AMPK at the specific subcellular compartments in cell behaviors is yet to be determined. Recent reviews suggested that compartment-specific regulation of AMPK might play a role in AMPK's final biological output, and subsequently cell behaviors [14, 41]. It has been reported that cancer cells use altered glucose metabolism at the mitochondria for energy homeostasis and survival $[42,43]$ and that mitochondria-targeted AMPK is involved in cell growth survival[44, 45]. We have also recently showed that AMPK in the mitochondria is activated by interstitial fluid flow in three-dimensional culture and that mitochondrial AMPK inhibition reduced cell migration and blocked flow-induced cell migration[17]. Given the context-dependent role for AMPK in cancer, which can exert pro- or anti-tumorigenic effects, there is a pressing need to investigate whether and how subcellular AMPK complexes contribute to AMPK-mediated downstream effects that may consequently determine the balance between tumor-suppressive or oncogenic AMPK signaling. 
Many studies reported that FAK and Src are the primary nodes in mechanotransduction signaling pathways[8, 23, 24, 46, 47]. However, little is known about their involvement in mechanotransduction of AMPK. Here we showed their potentially distinct roles in MDA-MB231 and MCF-10A cells. While all the subcellular AMPK activities tested were downregulated by the specific FAK and Src inhibitors in MDA-MB-231 cells, MCF-10A cells showed selective AMPK reduction under the inhibitors. We observed that some of the AMPK biosensors showed small FRET ratio changes under the FAK or Src inhibitor, although the results were statistically significant. While it is not clear whether these small changes would consequently affect cell behavior, previous studies have shown that the typical FRET ratio changes are in the range of $10-20 \%$ in response to extracellular stimuli that are known to affect cell behavior[13, 17, 23-25]. This range is consistent with our present study. The FAK and Src inhibitors blocked AMPK response to shear stress at the plasma membrane, nucleus, and mitochondria in MDA-MB-231 cells, whereas they only blocked AMPK at the nucleus in MCF-10A cells. Our data suggest that there may be differential FAK/Src-driven mechanosensitivity between cancer cells and normal cells[48]. Our data also indicate that shear stress-induced activities of Cyto- and Golgi-AMPK in MDA-MB-231 and MCF-10A were independent of FAK and Src. Other signaling proteins such as Piezos[49] or cellular structural integrity such as membrane fluidity[50] might be involved in this FAK/Src-independent AMPK activation by shear stress.

We observed in the present study that myosin II activity is essential for shear stress-induced AMPK activation. While incubation with other cytoskeletal drugs that specifically target actin filaments and microtubules partially inhibited subcellular AMPK activation under shear stress, ML-7 and blebbistatin that inhibit myosin II activity abrogated shear stress-induced AMPK 
activation regardless of subcellular AMPK locations. These results are consistent with a recent report suggesting that RhoA-ROCK-myosin II pathway is necessary for force-induced AMPK mechanotransduction[15]. Collectively, the cellular contractility pathway appears to play a critical role in force-induced AMPK activation[51].

Consistent with recent reports demonstrating the role of AMPK in the negative regulation of integrin $\beta 1[32,33]$, our data show that AMPK activation by A769662 significantly reduced the basal activity of FAK and Src. Together with the results in the present study on the regulation of AMPK by FAK and Src, there appears to be a feedback loop between FAK/Src and AMPK activities. However, our data suggest that although AMPK affects the basal level of FAK and Src, it does not play a critical role in shear stress-induced activation of FAK and Src. It is possible that AMPK-driven integrin $\beta 1$ inhibition is not sufficient to mediate mechanotransduction of FAK and Src and other integrins such as $\alpha_{v} \beta_{3}[52,53]$ might be involved in shear stress-induced activation of FAK and Src.

\section{Conclusions}

Our results show that while cytosolic AMPK, the majority AMPK pool, is activated by shear stress in MDA-MB-231 and MCF-10A cells, these two cells have different subcellular AMPK activation patterns, suggesting the different mechanoresponsive metabolic signaling between breast cancer cells and normal epithelial cells. Importantly, FAK and Src as well as the myosin II-dependent signaling pathway appear to be involved in subcellular AMPK mechanotransduction. In the future, it will be of interest to investigate how individual 
extracellular stimuli affects specific subcellular pool of AMPK, which in turn influences cancer cell behavior, such as cell survival, migration, proliferation, and autophagy.

\section{Acknowledgements}

We thank J. Zhang (University of California San Diego, USA) and T. Inoue (Johns Hopkins

University, USA) for the gift of the AMPK biosensors, and Y. Wang (University of California San Diego, USA) for the gift of the FAK and Src biosensors.

\section{Funding}

This work was supported in part by the funds from a breast cancer advocacy group, 100 Voices of Hope, and Biomechanics and Biomaterials Research Center - Integrated Nanosystems Development Institute at the Indiana University Purdue University Indianapolis.

\section{Conflicts of Interest}

None 


\section{References}

[1] D.T. Butcher, T. Alliston, V.M. Weaver, A tense situation: forcing tumour progression, Nat Rev Cancer 9(2) (2009) 108-22.

[2] D. Wirtz, K. Konstantopoulos, P.C. Searson, The physics of cancer: the role of physical interactions and mechanical forces in metastasis, Nat Rev Cancer 11(7) (2011) 512-22.

[3] A. Nagelkerke, J. Bussink, A.E. Rowan, P.N. Span, The mechanical microenvironment in cancer: How physics affects tumours, Semin Cancer Biol 35 (2015) 62-70.

[4] R.K. Jain, J.D. Martin, T. Stylianopoulos, The role of mechanical forces in tumor growth and therapy, Annu Rev Biomed Eng 16 (2014) 321-46.

[5] A.C. Shieh, M.A. Swartz, Regulation of tumor invasion by interstitial fluid flow, Phys Biol 8(1) (2011) 015012.

[6] L. Chin, Y. Xia, D.E. Discher, P.A. Janmey, Mechanotransduction in cancer, Curr Opin Chem Eng 11 (2016) 77-84.

[7] W. Guo, F.G. Giancotti, Integrin signalling during tumour progression, Nat Rev Mol Cell Biol 5(10) (2004) 816-26.

[8] S.K. Mitra, D.D. Schlaepfer, Integrin-regulated FAK-Src signaling in normal and cancer cells, Curr Opin Cell Biol 18(5) (2006) 516-23.

[9] X.H. Zhang, Q. Wang, W. Gerald, C.A. Hudis, L. Norton, M. Smid, J.A. Foekens, J. Massague, Latent bone metastasis in breast cancer tied to Src-dependent survival signals, Cancer Cell 16(1) (2009) 67-78.

[10] F.J. Sulzmaier, C. Jean, D.D. Schlaepfer, FAK in cancer: mechanistic findings and clinical applications, Nat Rev Cancer 14(9) (2014) 598-610. 
[11] D.G. Hardie, F.A. Ross, S.A. Hawley, AMPK: a nutrient and energy sensor that maintains energy homeostasis, Nat Rev Mol Cell Biol 13(4) (2012) 251-62.

[12] D. Garcia, R.J. Shaw, AMPK: Mechanisms of Cellular Energy Sensing and Restoration of Metabolic Balance, Mol Cell 66(6) (2017) 789-800.

[13] T. Miyamoto, E. Rho, V. Sample, H. Akano, M. Magari, T. Ueno, K. Gorshkov, M. Chen, H. Tokumitsu, J. Zhang, T. Inoue, Compartmentalized AMPK signaling illuminated by genetically encoded molecular sensors and actuators, Cell Rep 11(4) (2015) 657-70.

[14] A.S. Khan, D.E. Frigo, A spatiotemporal hypothesis for the regulation, role, and targeting of AMPK in prostate cancer, Nat Rev Urol 14(3) (2017) 164-180.

[15] J.L. Bays, H.K. Campbell, C. Heidema, M. Sebbagh, K.A. DeMali, Linking E-cadherin mechanotransduction to cell metabolism through force-mediated activation of AMPK, Nat Cell Biol 19(6) (2017) 724-731.

[16] A. Young, W. Wu, W. Sun, H. Benjamin Larman, N. Wang, Y.S. Li, J.Y. Shyy, S. Chien, G. Garcia-Cardena, Flow activation of AMP-activated protein kinase in vascular endothelium leads to Kruppel-like factor 2 expression, Arterioscler Thromb Vasc Biol 29(11) (2009) 1902-8. [17] H.E. Steele, Y. Guo, B.Y. Li, S. Na, Mechanotransduction of mitochondrial AMPK and its distinct role in flow-induced breast cancer cell migration, Biochem Biophys Res Commun $514(2)(2019)$ 524-529.

[18] T. Miyamoto, E. Rho, A. Kim, T. Inoue, Cellular Application of Genetically Encoded Sensors and Impeders of AMPK, Methods Mol Biol 1732 (2018) 255-272.

[19] Y. Wang, E.L. Botvinick, Y. Zhao, M.W. Berns, S. Usami, R.Y. Tsien, S. Chien, Visualizing the mechanical activation of Src, Nature 434(7036) (2005) 1040-5. 
[20] J. Seong, M. Ouyang, T. Kim, J. Sun, P.C. Wen, S. Lu, Y. Zhuo, N.M. Llewellyn, D.D. Schlaepfer, J.L. Guan, S. Chien, Y. Wang, Detection of focal adhesion kinase activation at membrane microdomains by fluorescence resonance energy transfer, Nat Commun 2 (2011) 406. [21] D.A. Zacharias, J.D. Violin, A.C. Newton, R.Y. Tsien, Partitioning of lipid-modified monomeric GFPs into membrane microdomains of live cells, Science 296(5569) (2002) 913-6. [22] D. Hanahan, R.A. Weinberg, Hallmarks of cancer: the next generation, Cell 144(5) (2011) 646-74.

[23] S. Na, O. Collin, F. Chowdhury, B. Tay, M. Ouyang, Y. Wang, N. Wang, Rapid signal transduction in living cells is a unique feature of mechanotransduction, Proc Natl Acad Sci U S A 105(18) (2008) 6626-31.

[24] Q. Wan, T. TruongVo, H.E. Steele, A. Ozcelikkale, B. Han, Y. Wang, J. Oh, H. Yokota, S. $\mathrm{Na}$, Subcellular domain-dependent molecular hierarchy of SFK and FAK in mechanotransduction and cytokine signaling, Sci Rep 7(1) (2017) 9033.

[25] Q. Wan, W. Xu, J.L. Yan, H. Yokota, S. Na, Distinctive subcellular inhibition of cytokineinduced SRC by salubrinal and fluid flow, PLoS One 9(8) (2014) e105699.

[26] N.O. Deakin, C.E. Turner, Distinct roles for paxillin and Hic-5 in regulating breast cancer cell morphology, invasion, and metastasis, Mol Biol Cell 22(3) (2011) 327-41.

[27] N. Balanis, M.K. Wendt, B.J. Schiemann, Z. Wang, W.P. Schiemann, C.R. Carlin, Epithelial to mesenchymal transition promotes breast cancer progression via a fibronectindependent STAT3 signaling pathway, J Biol Chem 288(25) (2013) 17954-67.

[28] B. Mezquita, P. Mezquita, M. Pau, L. Gasa, L. Navarro, M. Samitier, M. Pons, C. Mezquita, All-trans-retinoic acid activates the pro-invasive Src-YAP-Interleukin 6 axis in triple-negative 
MDA-MB-231 breast cancer cells while cerivastatin reverses this action, Sci Rep 8(1) (2018) 7047.

[29] X. Liu, R. Feng, Inhibition of epithelial to mesenchymal transition in metastatic breast carcinoma cells by c-Src suppression, Acta Biochim Biophys Sin (Shanghai) 42(7) (2010) 496501.

[30] J. Jian, Q. Yang, X. Huang, Src regulates Tyr(20) phosphorylation of transferrin receptor-1 and potentiates breast cancer cell survival, J Biol Chem 286(41) (2011) 35708-15.

[31] F.J. Alenghat, D.E. Ingber, Mechanotransduction: all signals point to cytoskeleton, matrix, and integrins, Sci STKE 2002(119) (2002) pe6.

[32] M. Georgiadou, J. Lilja, G. Jacquemet, C. Guzman, M. Rafaeva, C. Alibert, Y. Yan, P. Sahgal, M. Lerche, J.B. Manneville, T.P. Makela, J. Ivaska, AMPK negatively regulates tensindependent integrin activity, J Cell Biol 216(4) (2017) 1107-1121.

[33] E. Ross, R. Ata, T. Thavarajah, S. Medvedev, P. Bowden, J.G. Marshall, C.N. Antonescu, AMP-Activated Protein Kinase Regulates the Cell Surface Proteome and Integrin Membrane Traffic, PLoS One 10(5) (2015) e0128013.

[34] A. Katsumi, A.W. Orr, E. Tzima, M.A. Schwartz, Integrins in mechanotransduction, J Biol Chem 279(13) (2004) 12001-4.

[35] J. Zhang, M.D. Allen, FRET-based biosensors for protein kinases: illuminating the kinome, Mol Biosyst 3(11) (2007) 759-65.

[36] C.E. Antal, A.C. Newton, Spatiotemporal dynamics of phosphorylation in lipid second messenger signaling, Mol Cell Proteomics 12(12) (2013) 3498-508.

[37] P. Tsou, B. Zheng, C.H. Hsu, A.T. Sasaki, L.C. Cantley, A fluorescent reporter of AMPK activity and cellular energy stress, Cell Metab 13(4) (2011) 476-486. 
[38] Y. Kawai, Y. Yokoyama, M. Kaidoh, T. Ohhashi, Shear stress-induced ATP-mediated endothelial constitutive nitric oxide synthase expression in human lymphatic endothelial cells, Am J Physiol Cell Physiol 298(3) (2010) C647-55.

[39] T.C. Ju, H.M. Chen, J.T. Lin, C.P. Chang, W.C. Chang, J.J. Kang, C.P. Sun, M.H. Tao, P.H. Tu, C. Chang, D.W. Dickson, Y. Chern, Nuclear translocation of AMPK-alpha1 potentiates striatal neurodegeneration in Huntington's disease, J Cell Biol 194(2) (2011) 209-27.

[40] M. Kodiha, J.G. Rassi, C.M. Brown, U. Stochaj, Localization of AMP kinase is regulated by stress, cell density, and signaling through the MEK-->ERK1/2 pathway, Am J Physiol Cell Physiol 293(5) (2007) C1427-36.

[41] B. Faubert, E.E. Vincent, M.C. Poffenberger, R.G. Jones, The AMP-activated protein kinase (AMPK) and cancer: many faces of a metabolic regulator, Cancer Lett 356(2 Pt A) (2015) 16570.

[42] B. Chaube, P. Malvi, S.V. Singh, N. Mohammad, B. Viollet, M.K. Bhat, AMPK maintains energy homeostasis and survival in cancer cells via regulating p38/PGC-1alpha-mediated mitochondrial biogenesis, Cell Death Discov 1 (2015) 15063.

[43] R.C. Rabinovitch, B. Samborska, B. Faubert, E.H. Ma, S.P. Gravel, S. Andrzejewski, T.C. Raissi, A. Pause, J. St-Pierre, R.G. Jones, AMPK Maintains Cellular Metabolic Homeostasis through Regulation of Mitochondrial Reactive Oxygen Species, Cell Rep 21(1) (2017) 1-9. [44] J. Liang, Z.X. Xu, Z. Ding, Y. Lu, Q. Yu, K.D. Werle, G. Zhou, Y.Y. Park, G. Peng, M.J. Gambello, G.B. Mills, Myristoylation confers noncanonical AMPK functions in autophagy selectivity and mitochondrial surveillance, Nat Commun 6 (2015) 7926. 
[45] S. Jiang, Y. Wang, L. Luo, F. Shi, J. Zou, H. Lin, Y. Ying, Y. Luo, Z. Zhan, P. Liu, B. Zhu, D. Huang, Z. Luo, AMP-activated protein kinase regulates cancer cell growth and metabolism via nuclear and mitochondria events, J Cell Mol Med 23(6) (2019) 3951-3961.

[46] J.D. Humphrey, E.R. Dufresne, M.A. Schwartz, Mechanotransduction and extracellular matrix homeostasis, Nat Rev Mol Cell Biol 15(12) (2014) 802-12.

[47] H. Mohammadi, E. Sahai, Mechanisms and impact of altered tumour mechanics, Nat Cell Biol 20(7) (2018) 766-774.

[48] S.C. Lien, S.F. Chang, P.L. Lee, S.Y. Wei, M.D. Chang, J.Y. Chang, J.J. Chiu, Mechanical regulation of cancer cell apoptosis and autophagy: roles of bone morphogenetic protein receptor, Smad1/5, and p38 MAPK, Biochim Biophys Acta 1833(12) (2013) 3124-3133.

[49] B. Coste, J. Mathur, M. Schmidt, T.J. Earley, S. Ranade, M.J. Petrus, A.E. Dubin, A. Patapoutian, Piezo1 and Piezo2 are essential components of distinct mechanically activated cation channels, Science 330(6000) (2010) 55-60.

[50] B. Liu, S. Lu, Y.L. Hu, X. Liao, M. Ouyang, Y. Wang, RhoA and membrane fluidity mediates the spatially polarized Src/FAK activation in response to shear stress, Sci Rep 4 (2014) 7008.

[51] T. Isogai, J.S. Park, G. Danuser, Cell forces meet cell metabolism, Nat Cell Biol 19(6) (2017) 591-593.

[52] E. Puklin-Faucher, M.P. Sheetz, The mechanical integrin cycle, J Cell Sci 122(Pt 2) (2009) 179-86.

[53] A.W. Lambert, S. Ozturk, S. Thiagalingam, Integrin signaling in mammary epithelial cells and breast cancer, ISRN Oncol 2012 (2012) 493283. 


\section{Figure Legend}

Figure 1. AMPK activities at each subcellular compartment in response to fluid flow-induced shear stress in MDA-MB-231 cells. (a) Cyto-AMPK activities. 2.5 dyne $/ \mathrm{cm}^{2}, \mathrm{n}=11 ; 10$ dyne $/ \mathrm{cm}^{2}, \mathrm{n}=11$. (b) PM-AMPK activities. 2.5 dyne $/ \mathrm{cm}^{2}, \mathrm{n}=7 ; 10$ dyne $/ \mathrm{cm}^{2}, \mathrm{n}=9$. (c) Nuc-AMPK activities. 2.5 dyne $/ \mathrm{cm}^{2}, \mathrm{n}=10 ; 10$ dyne $/ \mathrm{cm}^{2}, \mathrm{n}=9$. (d) Mito-AMPK activities 2.5 dyne/cm², $\mathrm{n}=9$; 10 dyne $/ \mathrm{cm}^{2}, \mathrm{n}=14$. (e) Golgi-AMPK activities. 2.5 dyne $/ \mathrm{cm}^{2}, \mathrm{n}=14 ; 10$ dyne $/ \mathrm{cm}^{2}, \mathrm{n}=13$. Scale bars, $10 \mu \mathrm{m}$.

Figure 2. AMPK activities at each subcellular compartment in response to fluid flow-induced shear stress in MCF-10A cells. (a) Cyto-AMPK activities. 2.5 dyne $/ \mathrm{cm}^{2}, \mathrm{n}=8 ; 10 \mathrm{dyne} / \mathrm{cm}^{2}$, $\mathrm{n}=12$. (b) PM-AMPK activities. 2.5 dyne $/ \mathrm{cm}^{2}, \mathrm{n}=9 ; 10 \mathrm{dyne} / \mathrm{cm}^{2}, \mathrm{n}=9$. (c) Nuc-AMPK activities. 2.5 dyne $/ \mathrm{cm}^{2}, \mathrm{n}=10 ; 10 \mathrm{dyne} / \mathrm{cm}^{2}, \mathrm{n}=10$. (d) Mito-AMPK activities. 2.5 dyne $/ \mathrm{cm}^{2}, \mathrm{n}=8 ; 10$ dyne $/ \mathrm{cm}^{2}, \mathrm{n}=11$. (e) Golgi-AMPK activities. 2.5 dyne $/ \mathrm{cm}^{2}, \mathrm{n}=11 ; 10$ dyne $/ \mathrm{cm}^{2}, \mathrm{n}=12$. Scale bars, $10 \mu \mathrm{m}$.

Figure 3. AMPK activities at each subcellular compartment under PF573228 and PP2. (a) AMPK activities in response to PF573228 in MDA-MB231 cells. $n>8$. (b) AMPK activities in response to PP2 in MDA-MB231 cells. $n>10$. (c) AMPK activities in response to PF573228 in MCF-10A cells. $n>9$. (d) AMPK activities in response to PP2 in MCF-10A cells. $n>9$. Scale bars, $10 \mu \mathrm{m} . * \mathrm{p}<0.05, * * \mathrm{p}<0.01$, and $* * * \mathrm{p}<0.001$ compared to the corresponding FRET activities at $0 \mathrm{~min}$. 
Figure 4. The flow-driven activities of AMPK after pre-treatment with PF573228 and PP2. Cells transfected with one of the AMPK biosensors were pretreated with either $1000 \mathrm{nM}$ PF573228 or $10 \mu \mathrm{M}$ PP2 for 1 hour before application of fluid flow-induced shear stress. Each bar graph represents corresponding compartmentalized AMPK activities at 60 min that were normalized to those at 0 min. (a) AMPK activities in MDA-MB231 cells. Under 10 dyne/cm² + PF573228: Cyto-AMPK, n=9; PM-AMPK, n=10; Nuc-AMPK, n=13; Mito-AMPK, n=12; Golgi-AMPK, $\mathrm{n}=10$. Under 10 dyne/cm² + PP2: Cyto-AMPK, n=12; PM-AMPK, n=8; Nuc-AMPK, n=12; Mito-AMPK, n=14; Golgi-AMPK, n=10. (b) AMPK activities in MCF-10A cells. Under 10 dyne/cm² + PF573228: Cyto-AMPK, n=13; PM-AMPK, n=12; Nuc-AMPK, n=13; Mito-AMPK, n=15; Golgi-AMPK, n=13. Under 10 dyne/cm² + PP2: Cyto-AMPK, n=11; PM-AMPK, n=14; Nuc-AMPK, n=11; Mito-AMPK, n=14; Golgi-AMPK, n=11. The number of samples under 10 dyne $/ \mathrm{cm}^{2}$ alone in (a) and (b) is shown in Figs. 1 and 2, respectively. Scale bars, $10 \mu \mathrm{m} . *$ $\mathrm{p}<0.05, \bullet \mathrm{p}<0.01$, and $+\mathrm{p}<0.001$ compared to the corresponding FRET activities at 0 min.

Figure 5. The role of the cytoskeletal components in the regulation of shear stress-induced AMPK activities. Cells transfected with one of the AMPK biosensors were pretreated with one of the reagents (Cytochalasin D, Nocodazole, ML-7, or Blebbistatin) for 1 hour before FRET imaging. Each bar graph represents corresponding compartmentalized AMPK activities at 60 min that were normalized to those at 0 min. (a) Cyto-AMPK activity. (b) PM-AMPK activity. (c) Nuc-AMPK activity. (d) Mito-AMPK activity. (e) Golgi-AMPK activity. $\mathrm{n}=10 \sim 23$ cells. * $\mathrm{p}<0.05, * * \mathrm{p}<0.01, * * * \mathrm{p}<0.001$ compared to the control group. 
Figure 6. The role of AMPK in the activities of FAK and Src. (a) The basal level of FAK and Src activities of three different treatment groups in MDA-MB-231 cells. In the Lyn-FAK:

Control, $n=11$; A769662, n=16; Compound C, n=14. In the Lyn-Src: Control, n=14; A769662, $\mathrm{n}=13$; Compound $\mathrm{C}, \mathrm{n}=17$. (b) The basal level of FAK and Src activities of three different treatment groups in MCF-10A cells. In the Lyn-FAK: Control, n=28; A769662, n=17;

Compound C, n=16. In the Lyn-Src: Control, n=18; A769662, n=15; Compound C, n=18. (c-d) Mechanotransduction of FAK and Src under treatment of AMPK activator and inhibitor. Cells transfected with either Lyn-FAK or Lyn-Src were pretreated with either an AMPK activator (A769662) or inhibitor (Compound C) for 1 hour before FRET imaging. The bar graphs were normalized to the corresponding biosensor and treatment group at $0 \mathrm{~min}$. (c) FAK activities. In MDA-MB-231: shear stress alone, $\mathrm{n}=17$; shear stress + A769662, $\mathrm{n}=17$; shear stress + Compound C, n=14. In MCF-10A: shear stress alone, $n=20$; shear stress + A769662, $n=21$; shear stress + Compound $C, n=17$. (d) Src activities. In MDA-MB-231: shear stress alone, $n=11$; shear stress + A769662, n=13; shear stress + Compound C, n=13. In MCF-10A: shear stress alone, $\mathrm{n}=11$; shear stress + A769662, $\mathrm{n}=16$; shear stress + Compound $\mathrm{C}, \mathrm{n}=13$. Scale bars, $10 \mu \mathrm{m} . *$ $\mathrm{p}<0.05,{ }^{* *} \mathrm{p}<0.01,{ }^{* * *} \mathrm{p}<0.001$ compared to the control group in (a-b) and corresponding FRET activities at $0 \mathrm{~min}$ in (c-d). 
a
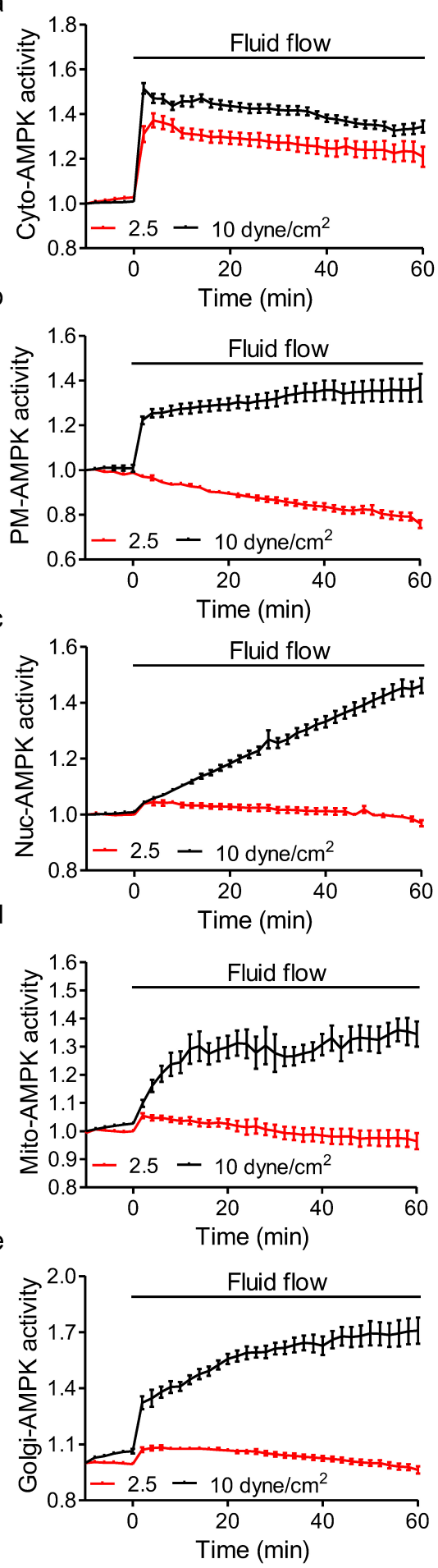
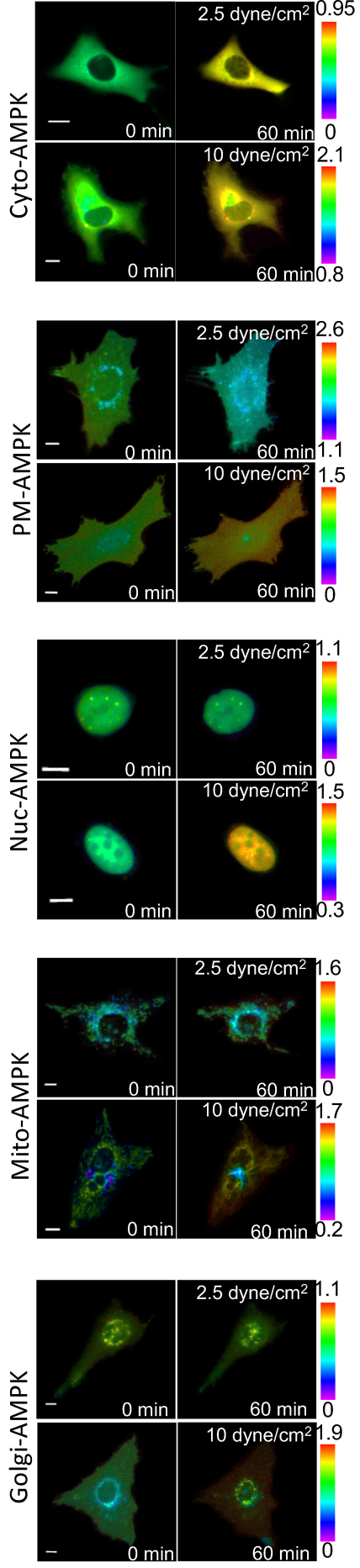
a
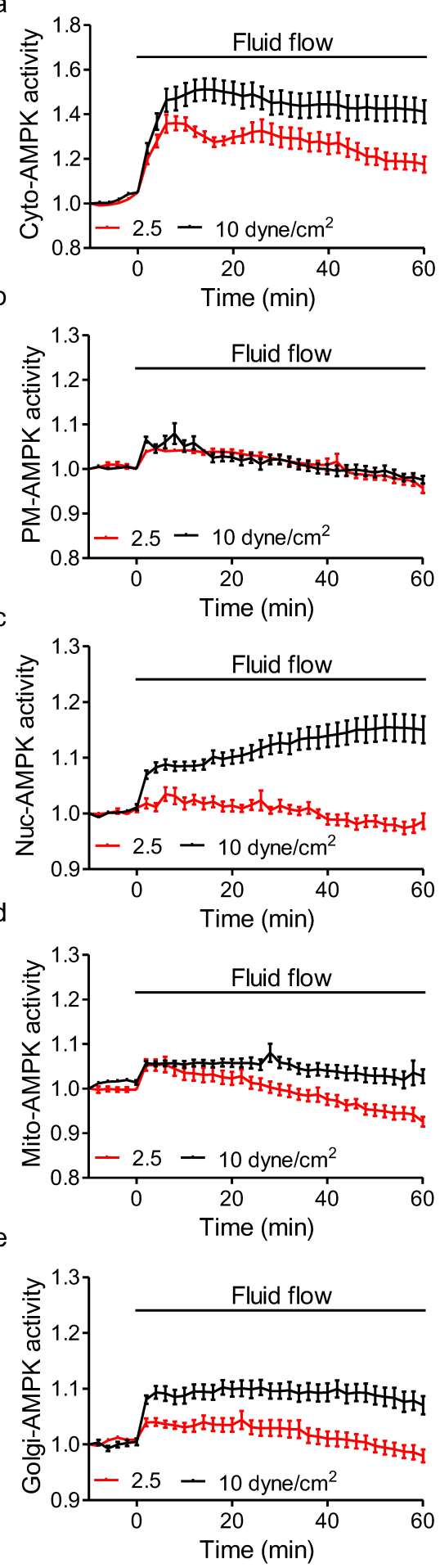
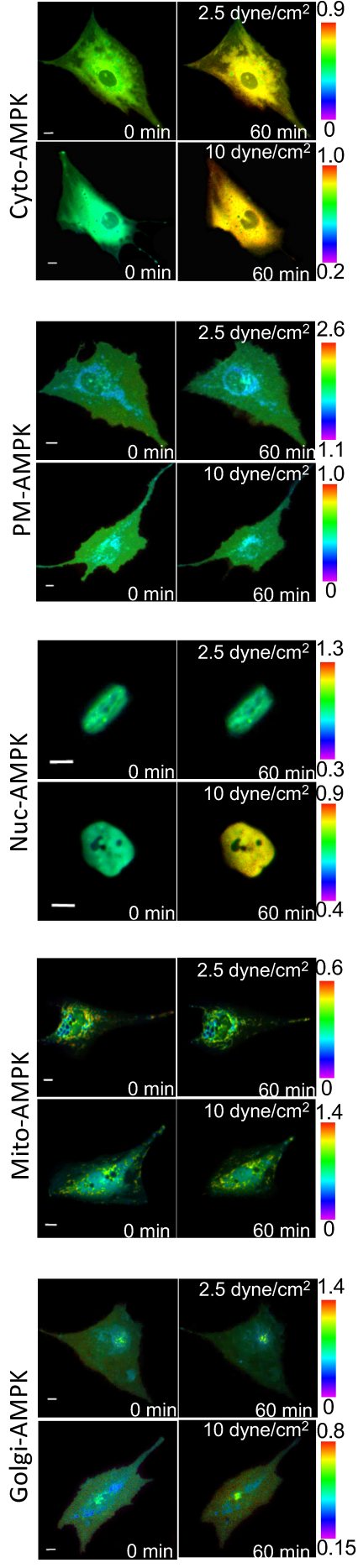
a
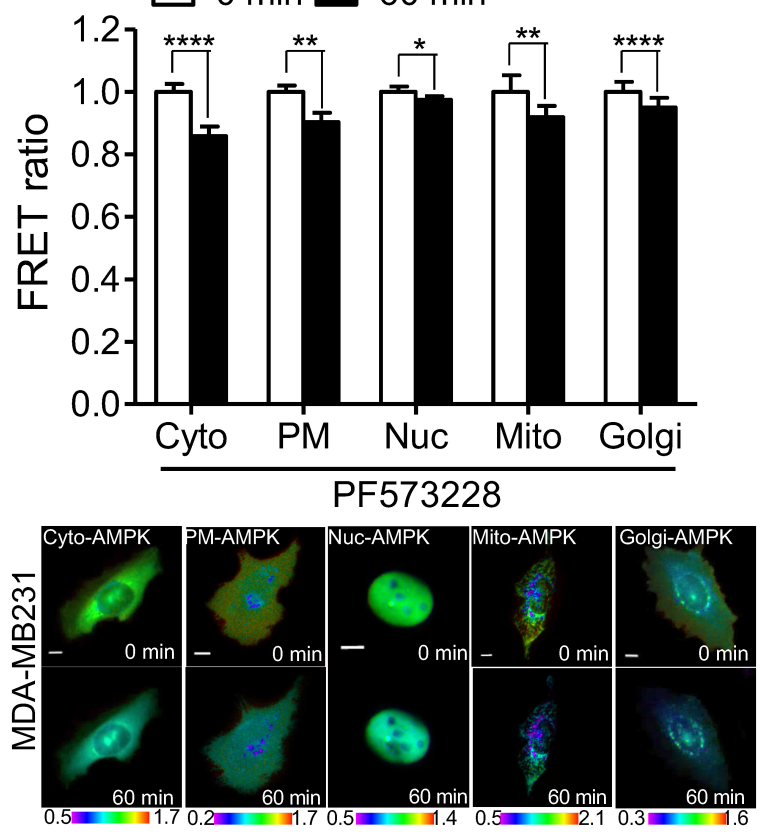

C
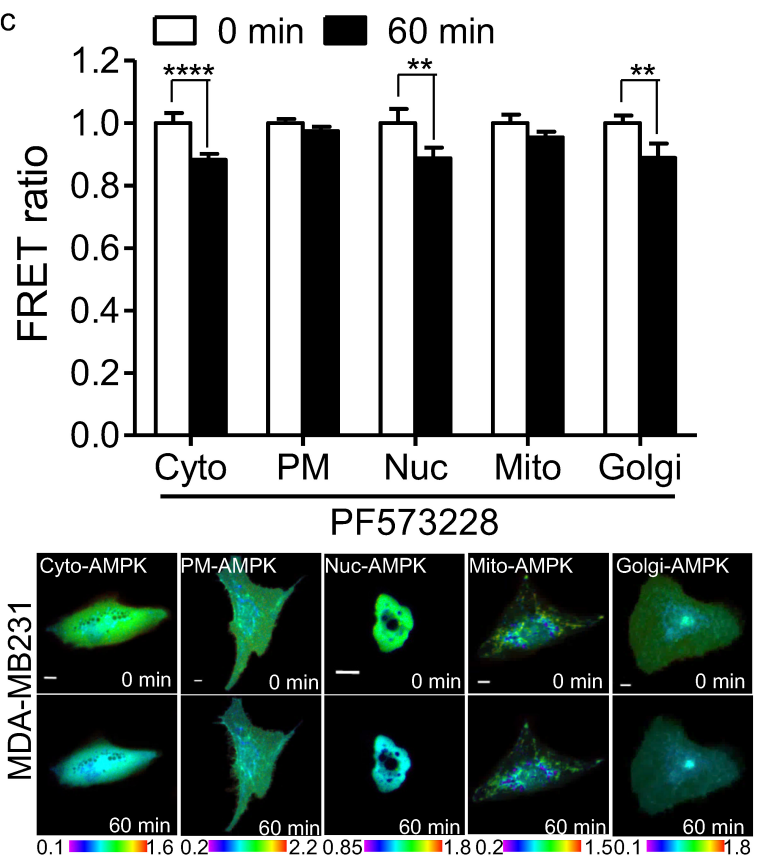

b
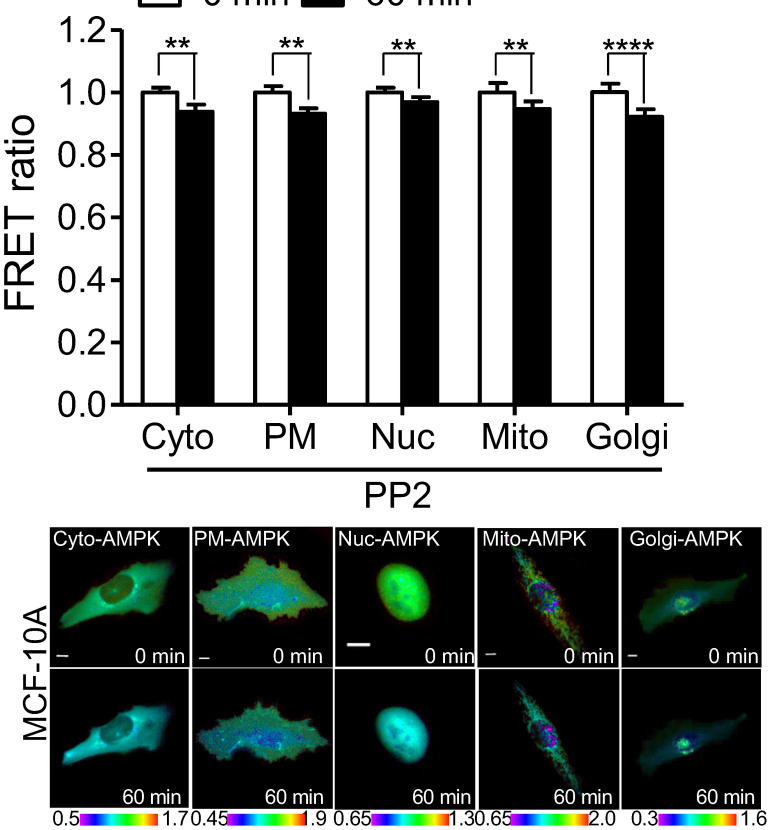

d $\square 0 \min \square 60$ min
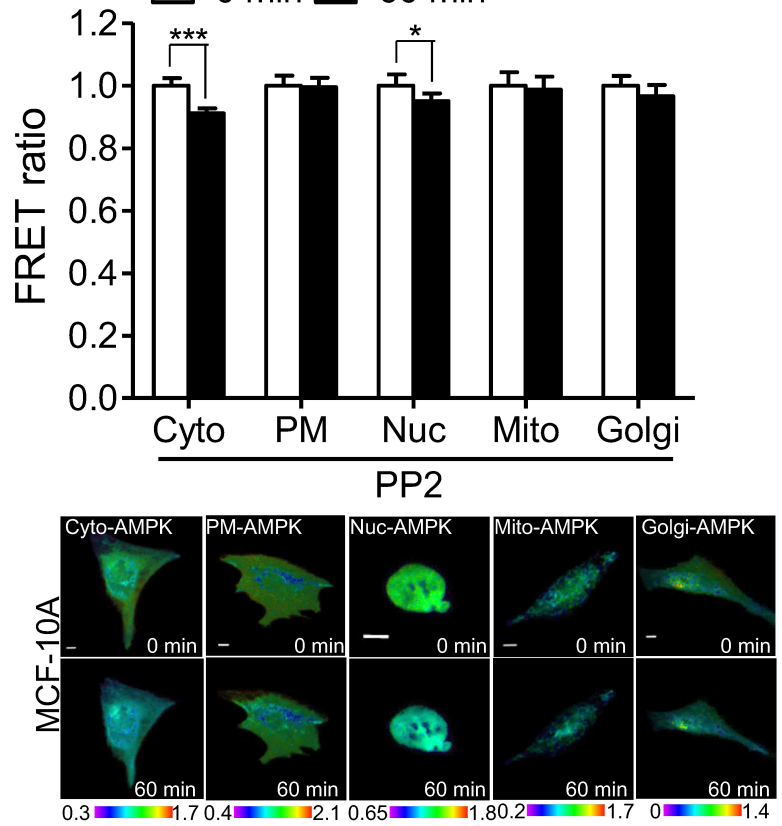

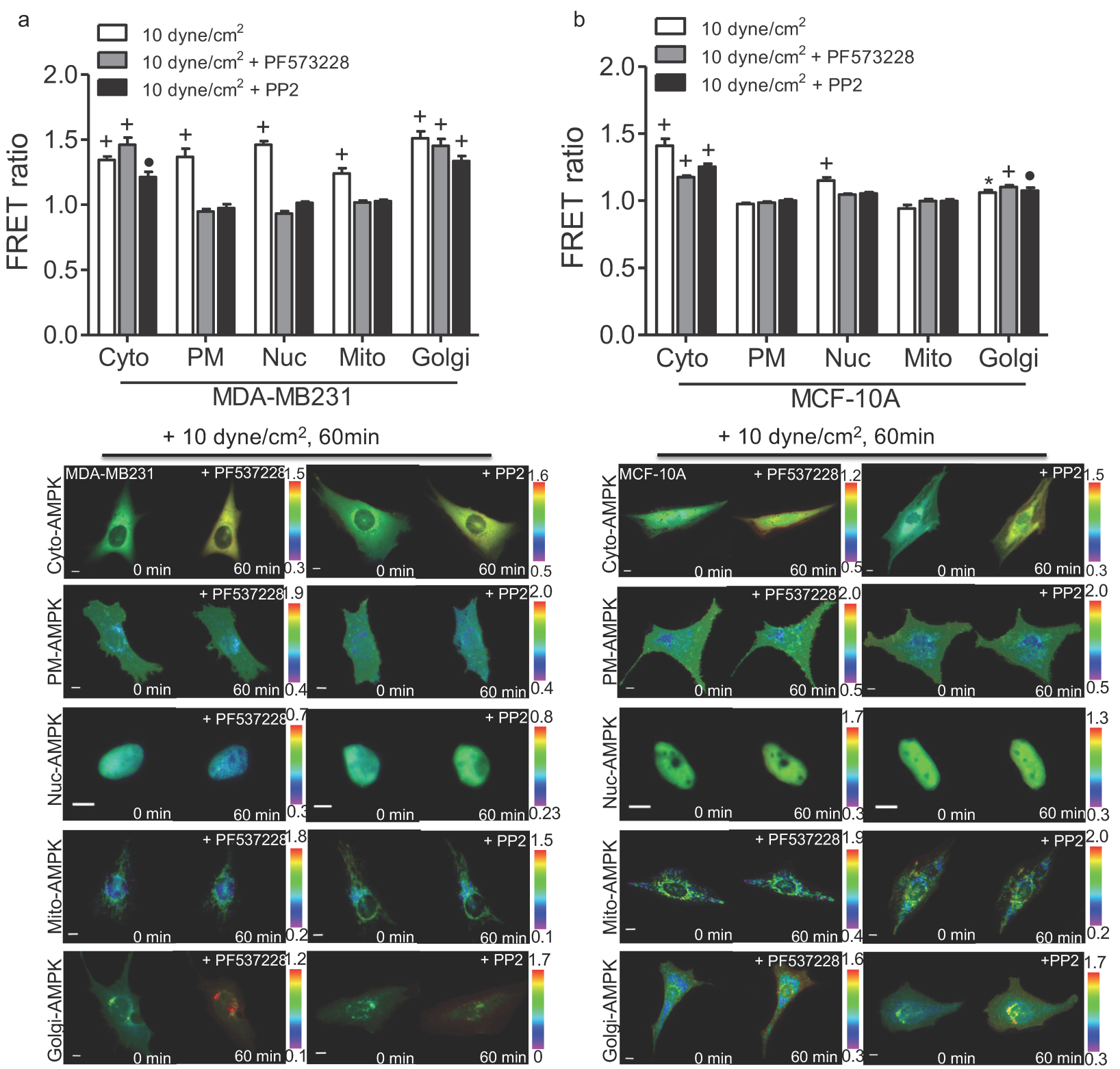

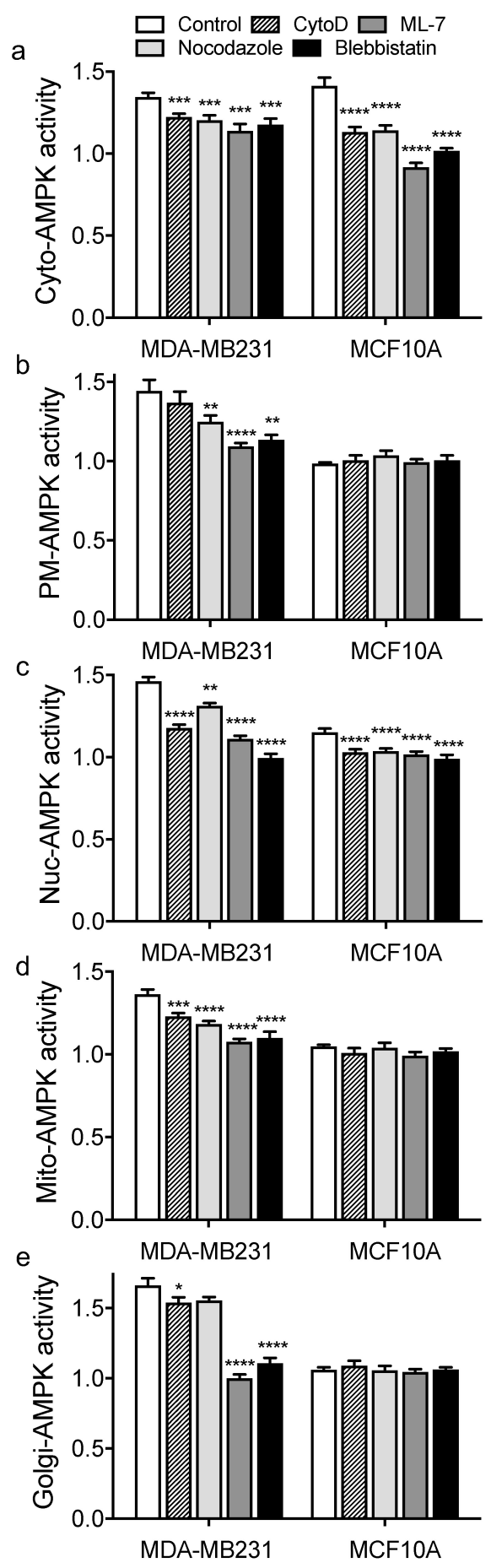

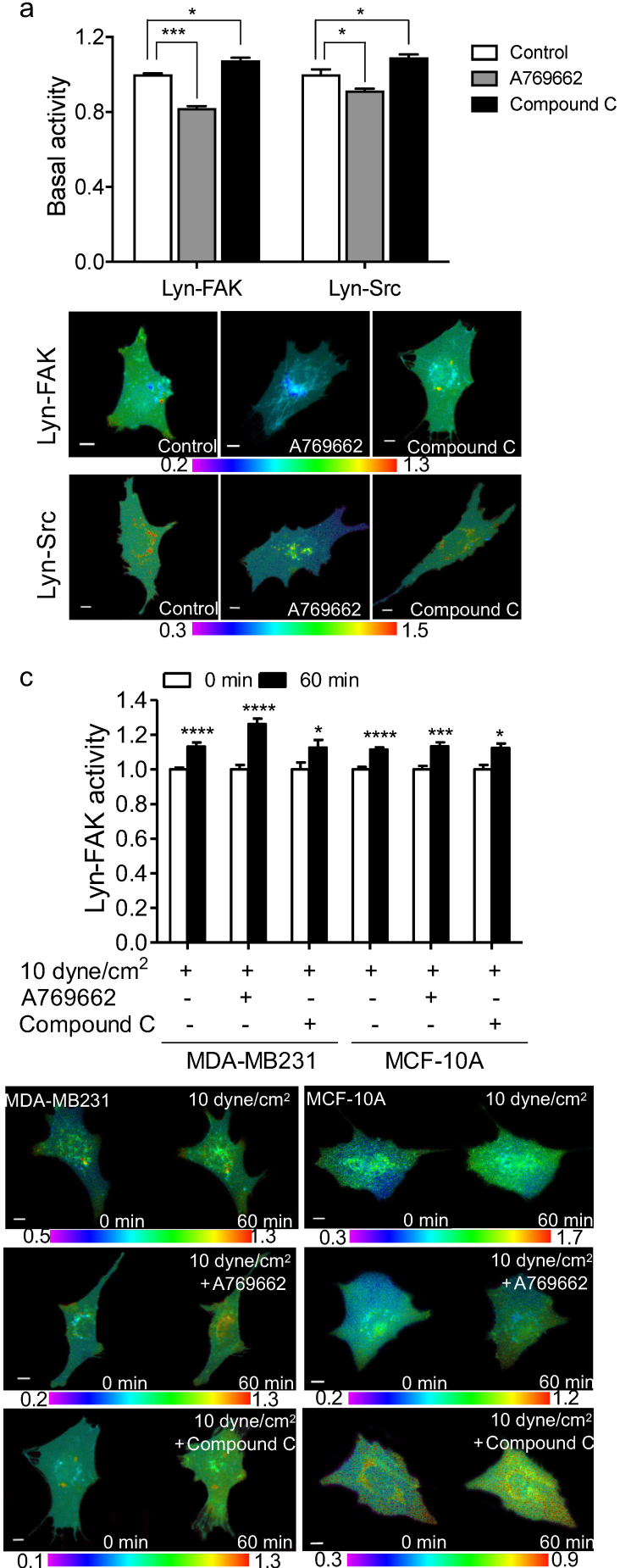

b
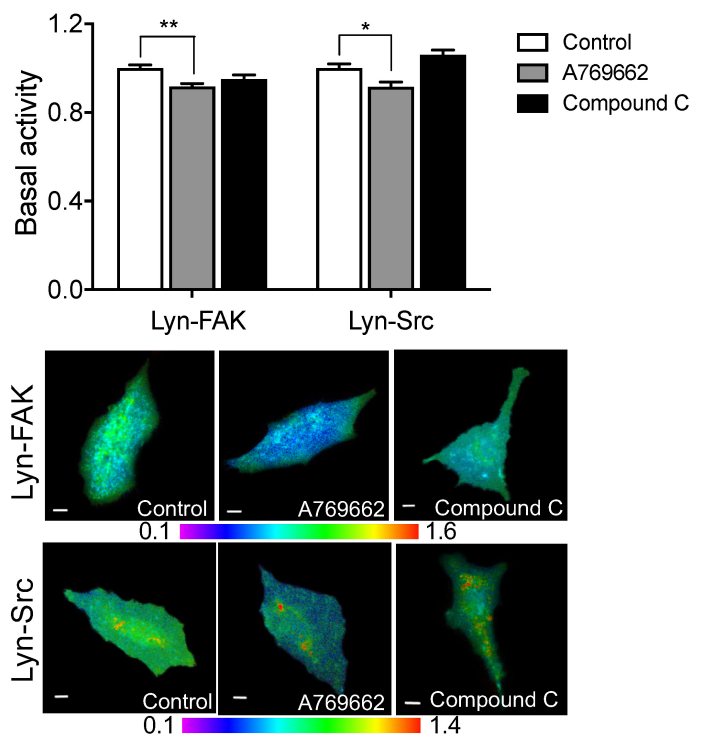

d
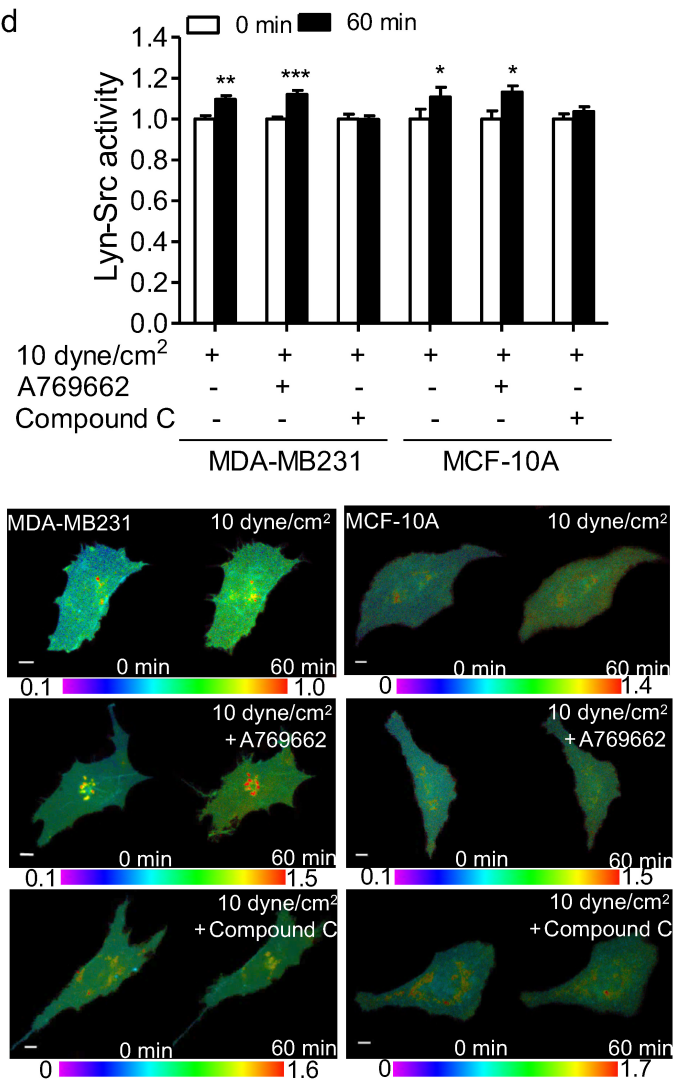


\section{Supplementary material}

Fluid flow-induced activation of subcellular AMPK and its interaction with FAK and SrC

Yunxia Guo ${ }^{1,2}$, Hannah E Steele ${ }^{1}$, Bai-Yan $\mathrm{Li}^{2 *}$, Sungsoo $\mathrm{Na}^{1^{*}}$

${ }^{1}$ Department of Biomedical Engineering, Indiana University Purdue University Indianapolis, Indianapolis, IN 46202, USA

${ }^{2}$ Department of Pharmacology, School of Pharmacy, Harbin Medical University, Harbin 150081, China
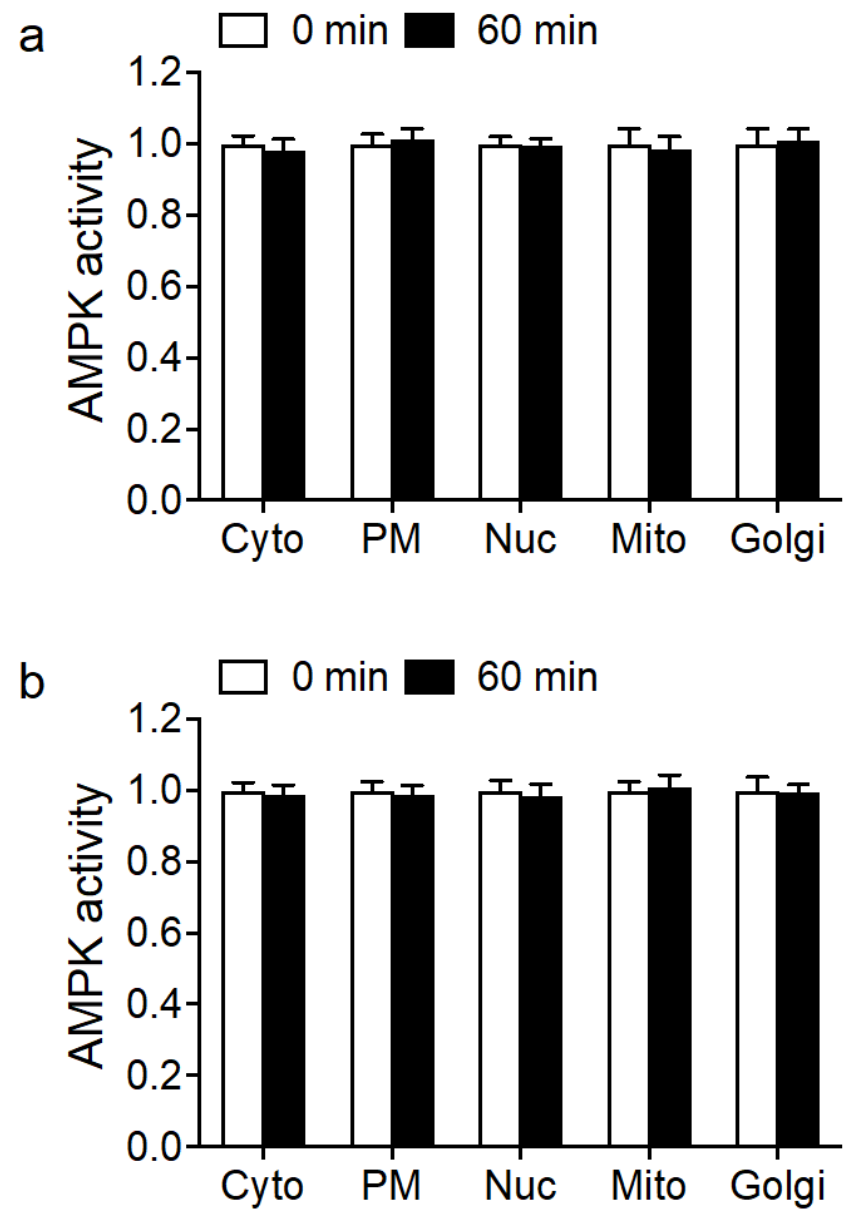

Figure S1. AMPK activities at each subcellular compartment under fluid flow-induced shear stress at 0 dyne $/ \mathrm{cm}^{2}$. (a) MDA-MB-231 cells. $\mathrm{n}>12$. (b) MCF-10A cells. $\mathrm{n}>10$. 

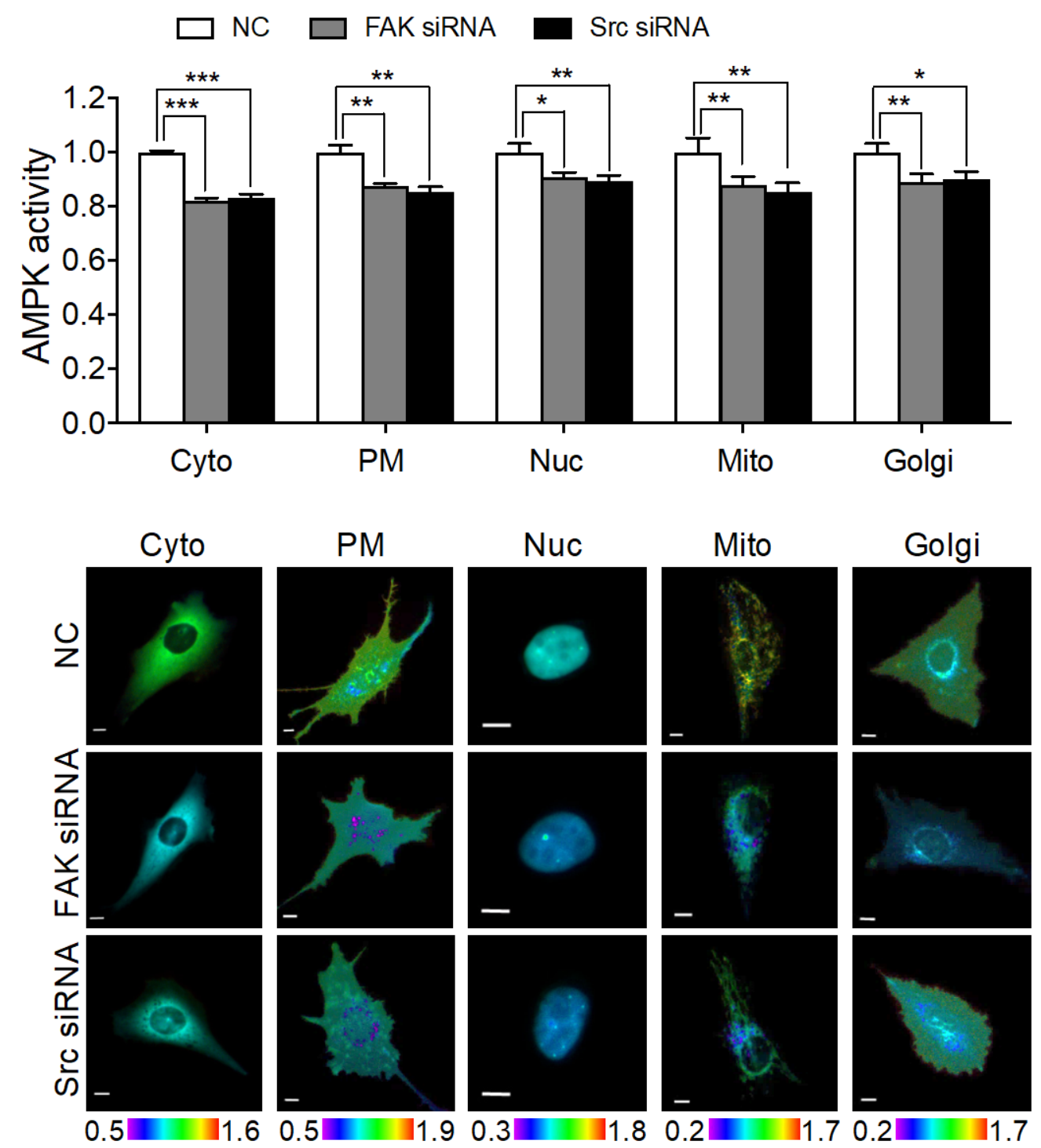

Figure S2. The basal level of AMPK activities at each subcellular compartment in MDA-MB231 cells transfected with negative control (NC), FAK siRNA, or Src siRNA. $n>12$. * $p<0.05$, ** $\mathrm{p}<0.01,{ }^{* * *} \mathrm{p}<0.001$. Scale bars, $10 \mu \mathrm{m}$. 